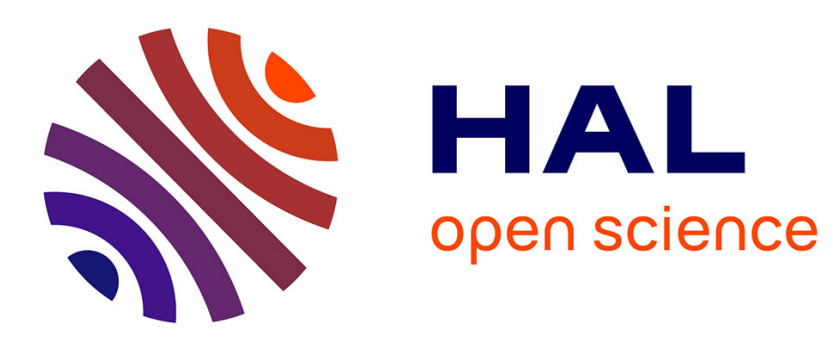

\title{
Filtres optiques à couplage de modes polarisés
}

J.P. Laurenti, P. Vaigot, M. Rouzeyre

\section{To cite this version:}

J.P. Laurenti, P. Vaigot, M. Rouzeyre. Filtres optiques à couplage de modes polarisés. Revue de Physique Appliquée, 1977, 12 (11), pp.1755-1766. 10.1051/rphysap:0197700120110175500 . jpa00244400

\section{HAL Id: jpa-00244400 https://hal.science/jpa-00244400}

Submitted on 1 Jan 1977

HAL is a multi-disciplinary open access archive for the deposit and dissemination of scientific research documents, whether they are published or not. The documents may come from teaching and research institutions in France or abroad, or from public or private research centers.
L'archive ouverte pluridisciplinaire HAL, est destinée au dépôt et à la diffusion de documents scientifiques de niveau recherche, publiés ou non, émanant des établissements d'enseignement et de recherche français ou étrangers, des laboratoires publics ou privés. 


\title{
REVUE DE PHYSIQUE APPLIQUÉE
}

\author{
Classification \\ Physics Abstracts \\ $07.60 \mathrm{~F}-42.10-42.80-78.20 \mathrm{Fm}$
}

FILTRES OPTIQUES A COUPLAGE DE MODES POLARISÉS

\author{
J. P. LAURENTI, P. VAIGot et M. ROUZEYRE
}

Centre d'Etudes d'Electronique des Solides (*)

LA 21, USTL, place E.-Bataillon 34060 Montpellier-Cedex, France

(Reçu le 5 juillet 1977, accepté le 2 août 1977)

\begin{abstract}
Résumé. - Nous décrivons le principe de fonctionnement de filtres optiques fonctionnant par transfert d'énergie entre deux modes électromagnétiques couplés polarisés à angle droit. Nous montrons que ce phénomène existe non seulement dans des matériaux à structure Wurtzite mais aussi dans des matériaux à structure zinc-blende. Ceci démontre que le couplage n'est pas spécifique à une classe cristalline et étend le domaine potentiel d'applications de l'ultra-violet, $\sim 1000 \AA$, au proche infrarouge. Les matériaux à structure Wurtzite et à forte dispersion de biréfringence tels CdS, $\mathrm{CdS}_{x} \mathrm{Se}_{1-x}$ et $\mathrm{Zn}_{1-x} \mathrm{Cd}_{x} \mathrm{~S}$ donnent des structures passe-bande ou coupe-bande extrêmement sélectives, $\Delta \lambda \sim 5$ à $10 \AA$ avec des taux de réjection hors-bande de l'ordre de $10^{3}$. La fréquence d'accord est soit fixe soit continuement accordable depuis $4300 \AA$ jusqu'à $7100 \AA$. Le facteur de transmission en bande passante atteint $80 \%$ à $77 \mathrm{~K}$ mais est réduit à $20 \%$ à $300 \mathrm{~K}$ par l'absorption résiduelle au voisinage du seuil d'absorption fondamentale. Dans les matériaux à structure zinc-blende, tel $\mathrm{ZnTe}$, l'amplitude de la biréfringence induite par compression est faible ce qui donne une largeur de bande de l'ordre de $100 \AA$. Cette largeur de bande peut toutefois être modifiée entre quelque 20 et $150 \AA$ en ajustant la valeur de la contrainte. A titre d'illustration nous terminons cet article par l'examen des performances limites d'une structure coupe-bande à $\mathrm{CdS}$, dont le taux de réjection atteint $75 \mathrm{db}$, susceptible d'être utilisée dans une application du type spectroscopie Raman.
\end{abstract}

\begin{abstract}
We describe the operating principles of optical filters using energie transfert between coupled orthogonally polarised electromagnetic waves. This phenomenon was previously demonstrated in Wurtzite type materials and is also shown to occur in piezo-birefringent zinc-blende crystals. This gives some evidence that the coupling does exist in many materials and manifests at the wavelength were the cancelation of the birefringence allows phase matching. This extends the spectral range of the applications from the UV, $1000 \AA$, to the near infra-red. Some experimental results have exhibited band-pass and cut-band features at fixed or continuously tunable frequencies. In Wurtzite type materials, $\mathrm{CdS}, \mathrm{CdS}_{x} \mathrm{Se}_{1-x}$ and $\mathrm{Zn}_{1-x} \mathrm{Cd}_{x} \mathrm{~S}$, the natural birefringence is strongly dispersive near the isotropic point and the selectivity is very high : $\Delta \lambda \sim 5 \AA$ at $77 \mathrm{~K}$ and $300 \mathrm{~K}$, while the rejection ratio is in the order of $10^{3}$. The effective transmission in the band pass region can be as large as $80 \%$ at $77 \mathrm{~K}$ but it reduces to $20 \%$ at room temperature due to residual absorption near the fundamental edge. With materials of the zinc-blende symmetry, such as $\mathrm{ZnTe}$, the main difference is that the magnitude of the piezo-birefringence is weak and this increases the band-with $\Delta \lambda$ to $\sim 100 \AA$. However, in this case, the bandwith can be varied between roughly $20 \AA$ to $150 \AA$ by adjusting the magnitude of the applied stress. Finally we investigate the potential of the application of a CdS cut-band filter, with a rejection ratio as large as $75 \mathrm{db}$, in Raman spectroscopy.
\end{abstract}

Introduction. - Le propos de cet article est double :

- Rappeler le principe de fonctionnement et faire connaître les performances de filtres optiques fonctionnant par couplage de modes électromagnétiques polarisés, du type passe-bande ou coupe-bande, à fréquence fixe ou continuement accordable.

- Rapporter un certain nombre de résultats originaux sur des cristaux à structure blende, qui démontrent la généralité du phénomène, étendent le

(*) Centre associé au C. N. R. S. domaine potentiel d'applications, et permettront peut-être de mieux comprendre le mécanisme microscopique responsable du transfert d'énergie entre modes polarisés.

Ce transfert d'énergie, entre modes E. M. polarisés à angle droit dans des cristaux biréfringents, a été mis en évidence en 1966 par C. H. Henry [1] dans des cristaux de CdS qui appartiennent à la classe $6 \mathrm{~mm}$. L'expérience la plus simple, figure 1 , consiste à placer un échantillon de quelques millimètres d'épaisseur entre nicols croisés, orientés selon les lignes neutres, et à éclairer en lumière blanche. Contrairement au 

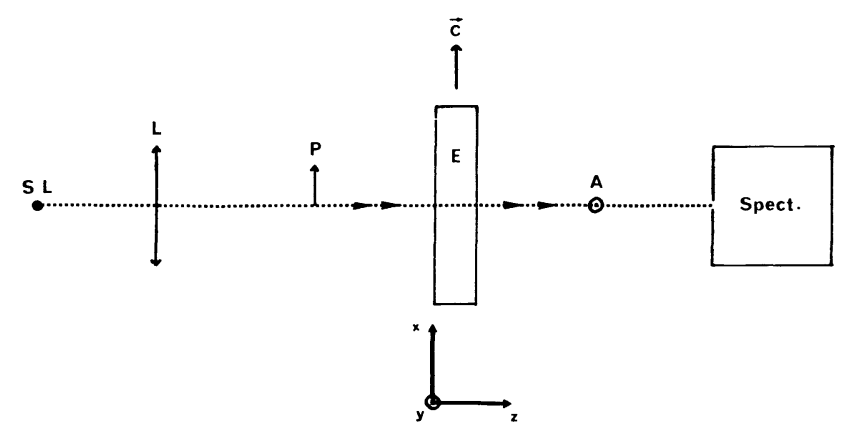

FIG. 1. - Schéma du montage: SL Source de Lumière; L Lentille de focalisation ; P Polariseur ; E Echantillon ; A Analyseur; Spect. Spectroscope.

[Experimental set up : SL Light Source ; L Lens ; P Polariser ; E Sample ; A Analyser ; Spect. Spectrometer.] résultat attendu, on observe un faisceau transmis dont l'analyse spectrale est reproduite sur la figure $2 a$.

Ce spectre de transmission présente un pic central de forte intensité, $t=\left(I_{t} / I_{0}\right) \simeq 20 \%$ à $300 \mathrm{~K}$ et $80 \%$ à $77 \mathrm{~K}$ avec une largeur au pied de quelque 5 à $10 \AA$. La longueur d'onde centrale est la longueur d'onde isotrope $\lambda_{i}$ qui correspond à l'annulation de la biréfringence naturelle du CdS. Le système fonctionne en filtre coupe-bande quand on fait tourner l'analyseur de $90^{\circ}$ pour se placer entre polariseurs parallèles, figure $2 b$. Il y a donc bien, pour la longueur d'onde $\lambda_{i}$, transfert d'énergie de la direction de polarisation rectiligne incidente vers une direction perpendiculaire.

L'interprétation phénoménologique donnée par C. H. Henry est celle d'un échange d'énergie par

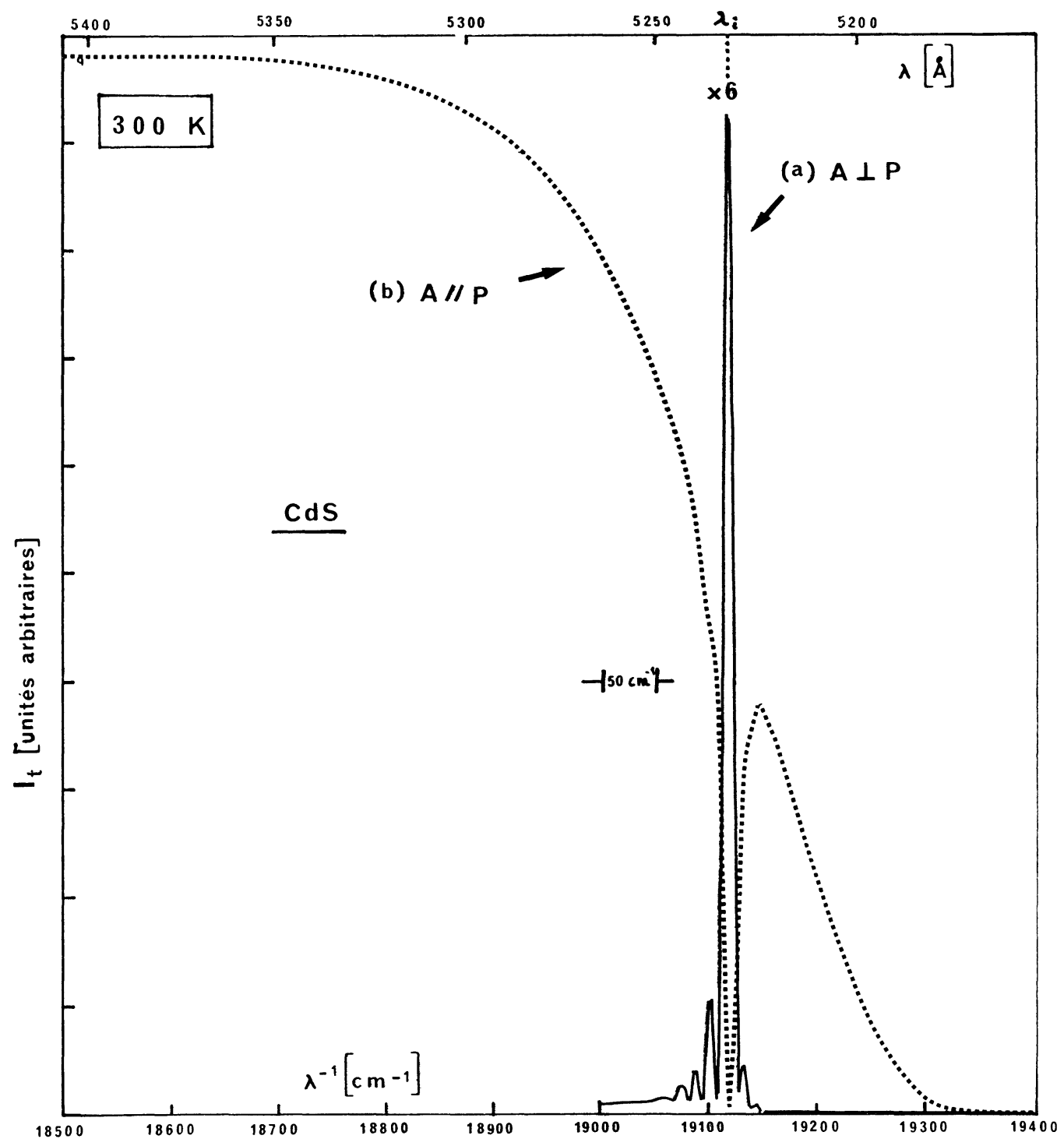

FIG. 2. - Analyse spectrale du faisceau transmis par un échantillon de CdS à $300 \mathrm{~K}$. La courbe en trait continu (a) est obtenue quand l'analyseur est perpendiculaire au polariseur, celle en trait pointillé $(b)$ quand l'analyseur est parallèle au polariseur. Le comportement global de cette dernière courbe est dû à l'absorption résiduelle au voisinage du gap.

[Spectral analysis of the transmitted light through a sample of CdS at $300 \mathrm{~K}$. The full line (a) corresponds to $A \perp P$, band-pass filter. The dotted line $(b)$ corresponds to $A / / P$, cut-band filter.] 
couplage entre les 2 oscillateurs spatiaux que constituent le mode ordinaire et le mode extraordinaire. Ce couplage n'est efficace que si ces oscillateurs sont spatialement isochrones c'est-à-dire que si l'accord de phase est réalisé. Nous résumerons cette description phénoménologique dans la section 1 de cet article en soulignant le rôle fondamental de la longueur de cohérence et les performances limites prévisibles par l'examen des courbes de dispersion de la biréfringence.

Ayant nous-mêmes accidentellement observé ce phénomène sur un cristal de $\mathrm{CdS}$ en 1973, et pris connaissance du travail de C. H. Henry, nous avons cherché à en étendre la portée sur le plan des applications potentielles et de la compréhension du mécanisme responsable du transfert d'énergie.

Notre premier objectif a été de réaliser des filtres optiques à fréquence fixe, ou mieux accordables, dans un domaine spectral aussi large que possible. Nous avons eu recours pour cela à des cristaux mixtes de $\mathrm{CdS}_{x} \mathrm{Se}_{1-x}$ et $\mathrm{Zn}_{x} \mathrm{Cd}_{1-x} \mathrm{~S}$ dont le gap varie régulièrement avec la composition chimique. C'est ainsi que la longueur d'onde d'absorption fondamentale varie de $3400 \AA$ environ dans $\mathrm{ZnS}$ à quelque $7100 \AA$ dans $\mathrm{CdSe}$. Nous pensions qu'il en serait de même pour la longueur d'onde isotrope $\lambda_{\mathbf{i}}$ et que le couplage se produirait dans ces matériaux qui appartiennent tous à la classe $6 \mathrm{~mm}$. C'est ce qu'ont pleinement confirmé nos premières expériences, [2] et [3], dont nous rappellerons l'essentiel dans la section 3 , après une brève description de l'appareillage et des cristaux utilisés dans la section 2 .

Notre deuxième objectif était de vérifier si le couplage entre modes polarisés à angle droit était spécifique à la structure Wurtzite ou s'il se produisait dans d'autres classes cristallines. Pour des raisons liées à l'appareillage et à l'approvisionnement en cristaux notre choix s'est porté sur le $\mathrm{ZnTe}$, structure blende.

P. Y. Yu and M. Cardona [4] ont étudié la piézobiréfringence de ce matériau; elle s'annule en changeant de signe vers $5700 \AA$ à $300 \mathrm{~K}$ et les résultats que nous rapportons dans la section 3 démontrent clairement que le couplage existe entre modes E. M. polarisés.

Pour terminer cet article il nous a paru intéressant, à titre d'illustration, d'étudier les performances d'une structure coupe-bande, utilisable par exemple pour éliminer la raie centrale de diffusion Rayleigh en spectroscopie Raman. Les résultats obtenus, $80 \mathrm{db}$ de réjection sur une largeur de $15 \mathrm{~cm}^{-1}$ autour de $20000 \mathrm{~cm}^{-1}$, semblent tout à fait prometteurs.

1. Interprétation phénoménologique du couplage entre modes E. M. polarisés. - Due à C. H. Henry [1] elle repose sur le mécanisme bien connu de transfert d'énergie entre 2 oscillateurs couplés qui sont ici le mode ordinaire et le mode extraordinaire dans un cristal anisotrope.

L'équation couplée de propagation s'obtient aisé- ment à partir des équations de Maxwell-Ampère et Maxwell-Faraday en introduisant la matrice représentative du tenseur de permittivité diélectrique : $\left[\varepsilon_{\mathrm{r}}\right]=\left[n^{2}\right]$ et celle de l'opérateur rotationnel : [rot], dans un système d'axes tri-rectangles.

On aboutit alors immédiatement à l'expression cherchée :

$$
\frac{4 \pi^{2}}{\lambda^{2}}\left[n^{2}\right]\left\{\begin{array}{c}
E_{x} \\
E_{y} \\
E_{z}
\end{array}\right\}=[\mathrm{rot}]^{2}\left\{\begin{array}{c}
E_{x} \\
E_{y} \\
E_{z}
\end{array}\right\} .
$$

1.1 EXEMPLE : ONDE PLANE AVEC PROPAGATION PERPENDICULAIRE A L'AXE D'UN CRISTAL UNIAXE. (Absorption et réflexions aux extrémités du cristal négligées). - Considérons à titre d'exemple la propagation d'une onde plane dans une direction $\mathrm{O} z$ perpendiculaire à l'axe optique d'un milieu uniaxe (Fig. 1). Cette dénomination suppose à priori que l'ellipsoïde des indices est de révolution autour de $\mathrm{O} x$. Admettons qu'il soit physiquement impossible de diagonaliser le tenseur $\left[\varepsilon_{\mathrm{r}}\right]$ parce qu'il est par exemple la somme de 2 tenseurs n'ayant pas le même système de vecteurs propres.

Il subsiste alors toujours un élément non diagona 1 $n_{x y}^{2}$ et l'équation de propagation devient :

$$
\frac{2 \pi^{2}}{\lambda^{2}}\left[\begin{array}{ll}
n_{\mathrm{e}}^{2} & n_{x y}^{2} \\
n_{x y}^{2} & n_{0}^{2}
\end{array}\right]\left\{\begin{array}{l}
E_{x} \\
E_{y}
\end{array}\right\}=k^{2}\left\{\begin{array}{c}
E_{x} \\
E_{y}
\end{array}\right\}
$$

qui redonne les solutions découplées usuelles,

$$
k_{\mathrm{e}}=\frac{2 \pi n_{\mathrm{e}}}{\lambda} \text { et } \quad k_{0}=\frac{2 \pi n_{0}}{\lambda}
$$

quand le couplage est nul, $n_{x y}=0$.

Pour résoudre $\left(\mathrm{I}^{\prime}\right)$ il est utile de faire une remarque concernant les ordres de grandeur des trois indices utilisés.

$$
\begin{aligned}
& n_{\mathrm{e}}=\left(1+\chi_{\mathrm{e}}\right)^{1 / 2} \sim 1 \\
& n_{0}=\left(1+\chi_{0}\right)^{1 / 2} \sim 1 \\
& n_{x y}=\left(\chi_{x y}\right)^{1 / 2} \sim 0_{+}
\end{aligned}
$$

$\chi=$ susceptibilité diélectrique

et d'introduire l'indice moyen :

$$
n=\frac{n_{\mathrm{e}}+n_{0}}{2} .
$$

On obtient alors pour les modes propres deux solutions :

$$
k_{+}=\frac{2 \pi n_{+}}{\lambda} \text { et } \quad k_{-}=\frac{2 \pi n_{-}}{\lambda}
$$

avec :

$$
n_{+}-n_{-}=\frac{\left(n_{x y}\right)^{2}}{n} \sqrt{1+Y^{2}} .
$$

Le scalaire $Y$, défini par :

$$
Y=\frac{n}{n_{x y}} \frac{n_{\mathrm{e}}-n_{0}}{n_{x y}}
$$


varie très rapidement autour de $\lambda_{\mathrm{i}}$. Si on prend par exemple $n_{x y}=10^{-3}, Y$ passe de la valeur 0 à la valeur

$$
\frac{n}{n_{x y}} \simeq 10^{3} \text { quand } \frac{n_{\mathrm{e}}-n_{0}}{n_{x y}}
$$

passe de la valeur 0 à la valeur 1 c'est-à-dire quand $n_{\mathrm{e}}-n_{0}$ passe de 0 à $10^{-3}$.

- Longueur de cohérence. - Pour une valeur donnée de $n_{x y}$, la distance entre vecteurs d'ondes :

$$
\begin{aligned}
k_{+}-k_{-}=\frac{2 \pi}{\lambda}\left(n_{+}-n_{-}\right)= & \frac{2 \pi}{L_{\mathrm{c}}}= \\
& =\frac{2 \pi}{\lambda} \frac{\left(n_{x y}\right)^{2}}{n} \sqrt{1+Y^{2}}
\end{aligned}
$$

est d'autant plus grande que la biréfringence $n_{\mathrm{e}}-n_{0}$ est plus forte. Ceci signifie que la fréquence du battement spatial des modes couplés croît avec le déphasage entre mode ordinaire et mode extraordinaire. $\mathrm{La}$ période spatiale du battement ou longueur de cohérence :

$$
L_{\mathrm{c}}=\frac{\lambda}{n_{+}-n_{-}}
$$

est maximale et égale à :

$$
L_{\mathrm{c}_{\mathrm{i}}}=\lambda_{\mathrm{i}} \frac{n}{n_{x y}} \cdot \frac{1}{n_{x y}}
$$

quand $n_{\mathrm{e}}-n_{0}=0$.

En négligeant la variation de $\lambda$ dans l'expression de la longueur de cohérence, on peut encore écrire :

$$
L_{\mathrm{c}(\lambda)}=\frac{L_{\mathrm{c}_{1}}}{\sqrt{1+Y^{2}}} .
$$

Cette dernière formule, compte tenu des remarques précédentes sur la variation de $Y$ autour de $\lambda_{\mathrm{i}}$, souligne la décroissance très rapide de la longueur de cohérence $L_{\mathrm{c}}$ dès que les 2 oscillateurs ne sont plus isochrones. C'est là l'explication de la très grande sélectivité de ces structures. Cette sélectivité croît avec la dispersion de la biréfringence autour de $\lambda_{i}$ c'est-à-dire avec la valeur de l'angle entre les courbes de dispersion de l'indice ordinaire et de l'indice extraordinaire au point d'intersection.

- Taux de commutation en énergie à l'abscisse z. En ajoutant les conditions initiales propres au cas de la figure 1 , c'est-à-dire :

$$
\begin{aligned}
& E_{x}=1 \\
& E_{y}=0
\end{aligned} \quad \text { en } z=0
$$

on établit aisément qu'à la profondeur $z$ le rapport des énergies transportées par la composante $E_{y}$ et par la composante $E_{x}$, désigné par taux de commutation, $T_{\mathrm{c}(\lambda)}$ est :

$$
T_{\mathrm{c}(\lambda)}=\frac{\left|E_{y}^{2}\right|}{\left|E_{x}^{2}\right|}=\frac{1}{1+Y_{(\lambda)}^{2}} \sin ^{2} \frac{\pi z}{L_{\mathrm{c}(\lambda)}} .
$$

$\mathrm{Si}$, par exemple, l'épaisseur du cristal est égale à la moitié de la longueur de cohérence isotrope, $e=L_{\mathrm{c}_{\mathrm{i}}} / 2$, le transfert en énergie est égal à 1 à la sortie du cristal pour $\lambda=\lambda_{\mathrm{i}}$.

Ce transfert est nul pour toutes les longueurs d'ondes telles que :

$$
e=p \cdot L_{\mathrm{c}(\lambda)}(p \text { entier })
$$

et présente des extrémas latéraux d'amplitude $\frac{1}{1+Y_{(\lambda)}^{2}}$ pour les longueurs d'ondes telles que :

$$
e=(2 p+1) \frac{L_{\mathrm{c}(\lambda)}}{2}(p \text { entier })
$$

- Largeur du pic central. - Pour préciser quantitativement la largeur du pic central, il suffit de se reporter aux courbes représentatives de :

$$
\frac{L_{\mathrm{c}(\lambda)}}{L_{\mathrm{c}_{\mathrm{i}}}}=\frac{1}{\sqrt{1+Y_{(\lambda)}^{2}}} \text { et } T_{\mathrm{cmax}}=\frac{1}{1+Y_{(\lambda)}^{2}} \quad \text { (Fig. 3). }
$$

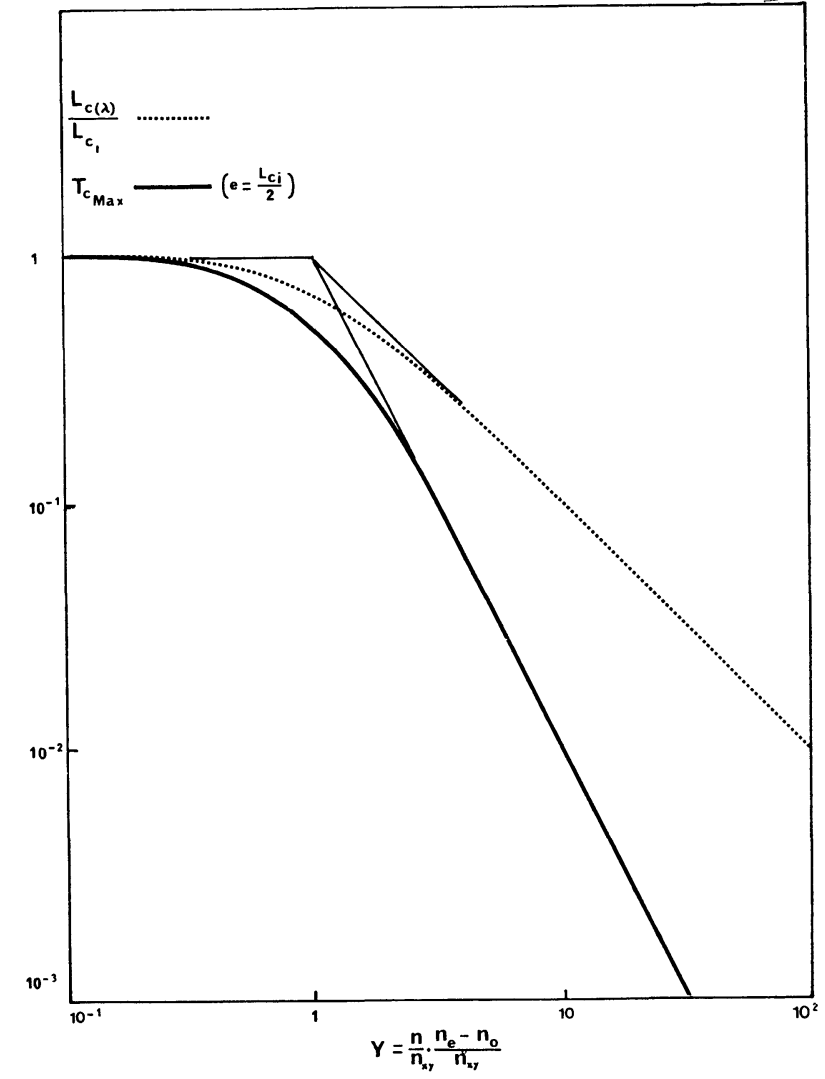

FIG. 3. - Variations de $L_{\mathrm{c}(\lambda)} / L_{\mathrm{c}_{\mathrm{i}}}$ (en trait pointillé) et de $T_{\mathrm{cmax}}$ (en trait continu) en fonction de la quantité

$$
Y=\frac{n}{n_{x y}} \cdot \frac{n_{\mathrm{e}}-n_{0}}{n_{x y}} .
$$

Les segments de droite représentent le comportement asymptotique.

[Variations of $L_{\mathrm{c}(\lambda)} / L_{\mathrm{ci}}$ (dotted line) and $T_{\mathrm{cmax}}$ (full line) as a function of the dimensionless parameter :

$$
Y=\frac{n}{n_{x y}} \frac{n_{\mathrm{e}}-n_{0}}{n_{x y}} .
$$

The asymptotic behaviors are shown by the straigth-lines]. 
Le comportement asymptotique de ces deux fonctions est familier aux électroniciens et présente des pentes caractéristiques de $20 \mathrm{db} /$ décade et $40 \mathrm{db} /$ décade respectivement. Partant de $n_{\mathrm{e}}-n_{0}=0$ c'est-à-dire $Y=0$ avec $e=L_{\mathrm{c}_{\mathrm{i}}} / 2$ par exemple, le premier minimum nul s'obtient quand la longueur de cohérence est divisée par 2 c'est-à-dire pour :

$$
\pm\left(n_{\mathrm{e}}-n_{0}\right)=\frac{1,7}{n}\left(n_{x y}\right)^{2} \sim\left(n_{x y}\right)^{2}
$$

La sélectivité du système autour de $\lambda_{\mathbf{i}}$ est donc, pour une valeur donnée de $n_{x y}$, d'autant meilleure que la biréfringence $n_{\mathrm{e}}-n_{0}$ varie plus vite autour de $\lambda_{i}$. En première approximation, on peut écrire autour de $\lambda_{\mathbf{i}}$ :

$$
\left(n_{\mathrm{e}}-n_{0}\right)_{\lambda_{\mathrm{i}}+\Delta \lambda}=\frac{\mathrm{d}\left(n_{\mathrm{e}}-n_{0}\right)}{\mathrm{d} \lambda} \Delta \lambda
$$

c'est-à-dire que la largeur du pic central est inversement proportionnelle à la dispersion de biréfringence $\frac{\mathrm{d}\left(n_{\mathrm{e}}-n_{0}\right)}{\mathrm{d} \lambda}$.

Réciproquement, quand on connaît la dispersion de biréfringence autour de $\lambda_{i}$, l'étude du pic de transmission permet de déterminer la valeur de l'indice non diagonal $n_{x y}$ et celle de la longueur de cohérence isotrope $L_{\mathrm{ci}}$. Considérons à titre d'exemple le cas particulier du CdS et plaçons-nous du côté des grandes longueurs d'ondes pour que l'absorption et les réflexions aux extrémités du cristal puissent être raisonnablement négligées. La dispersion de biréfringence naturelle du CdS a été étudiée notamment par S. J. Czyzak et al. [5] dont nous avons reproduit les résultats sur la figure 4. Autour de la longueur d'onde isotrope, la dispersion de biréfringence est :

$$
\frac{\mathrm{d}\left(n_{\mathrm{e}}-n_{0}\right)}{\mathrm{d} \lambda} \simeq 10^{-4} \AA^{-1} \text { à } 300 \mathrm{~K} \text {. }
$$

Expérimentalement la distance entre $\lambda_{\mathrm{i}}$ et le $1^{\circ} \mathrm{mini}-$ mum nul est à cette température de l'ordre de $5 \AA$. Il correspond à

$$
n_{\mathrm{e}}-n_{0}=\frac{1,7}{n}\left(n_{x y}\right)^{2}=5 \times 10^{-4} .
$$

Avec $n=2,5$, on obtient :

$$
\left(n_{x y}\right)^{2} \simeq 7 \times 10^{-4} \text { et } L_{\mathbf{c}_{i}} 1,7 \mathrm{~mm} .
$$

Cette dernière valeur est en parfait accord avec nos observations et celles de C. H. Henry puisque l'épaisseur des cristaux utilisés est de l'ordre du millimètre.

- Maximas latéraux. - Reportons-nous à nou-

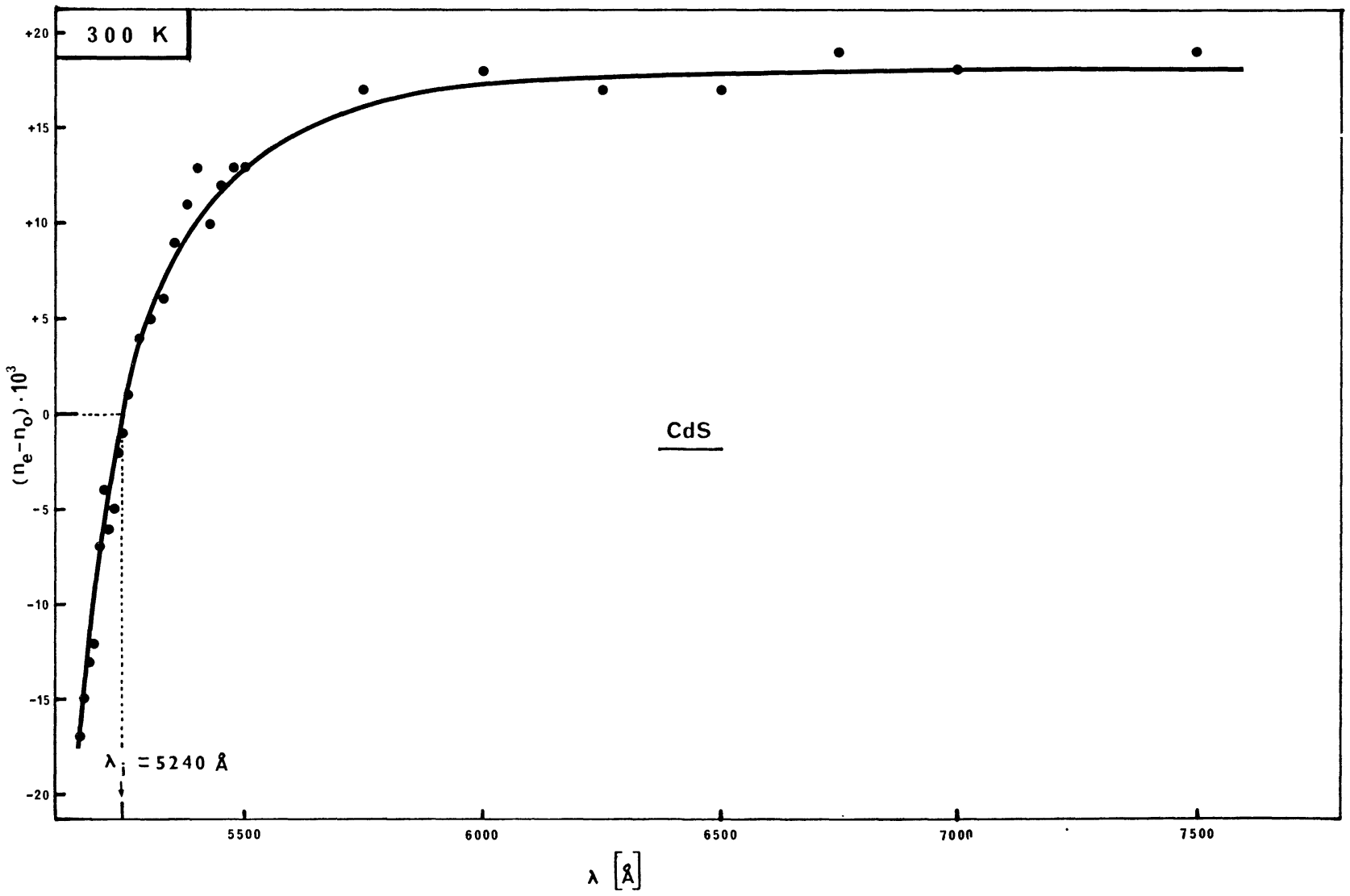

Fig. 4. - Dispersion de biréfringence naturelle du CdS à $300 \mathrm{~K}$ d'après S. J. Czyzak et al. [5]. Nous avons reporté les points expérimentaux.

[Dispersion of the natural birefringence of CdS at $300 \mathrm{~K}$ (from S. J. Czyzak and al. [5]).] 
veau aux courbes de la figure 3 . Le $1^{\circ}$ maximum latéral est obtenu pour :

$$
\frac{L_{\mathrm{c}(\lambda)}}{L_{\mathrm{c}_{\mathrm{i}}}}=\frac{1}{3} \quad \text { soit } \quad \pm Y=2,83
$$

Son amplitude relative est $1 / 9$. Les maximas latéraux (et minimas) sont quasi équidistants en $\left(n_{\mathrm{e}}-n_{0}\right)$ mais pas en longueur d'onde car la pente $\mathrm{d}\left(n_{\mathrm{e}}-n_{0}\right) / \mathrm{d} \lambda$ varie vite autour de $\lambda_{\mathbf{i}}$.

Précisons également que l'examen de la courbe de dispersion de la biréfringence permet de fixer le rapport entre l'amplitude du pic central et celle d'un pic latéral situé très loin de $\lambda_{\mathbf{i}}$.

En effet la biréfringence $n_{\mathrm{e}}-n_{0}$ du CdS tend vers une valeur limite de l'ordre de :

$$
\left(n_{\mathrm{e}}-n_{0}\right)_{1 \mathrm{im}}=20 \times 10^{-3} .
$$

Avec $\left(n_{x y}\right)^{2}=7 \times 10^{-4}$ et $n=2,5$ cela correspond à une valeur maximale de $Y$ de l'ordre de $10^{2}$ et à un affaiblissement maximum de 80 décibels entre le pic central et un pic satellite situé loin de $\lambda_{\mathbf{i}}$.

On peut donc, par le simple examen des courbes de dispersion de la biréfringence, prévoir les performances limites d'une telle structure passe-bande (idem pour le coupe-bande).

\subsection{INFLUENCE DE L'OUVERTURE DU FAISCEAU LUMI-} NEUX. - Ce que nous venons de rapporter reste vrai pour toute direction de propagation perpendiculaire à l'axe optique et l'ouverture du faisceau dans le plan perpendiculaire à l'axe n'altère en rien les performances.

L'ouverture du faisceau dans un plan contenant l'axe optique produit une diminution de l'efficacité $\mathrm{du}$ transfert mais cette diminution est faible puisque la composante utile varie en $\cos \theta$ et le transfert en $\cos ^{2} \theta$. Il y a donc là un avantage certain de ce type de filtres sur les filtres interférentiels.

1.3 INFLUENCE DE L'ABSORPTION ET DES RÉFLEXIONS AUX EXTRÉMITÉS. - Plutôt que d'effectuer la résolution exacte, et sans difficultés, du système d'équations couplées $(I)$ en introduisant des indices complexes, nous prendrons simplement en compte l'absorption et les réflexions sur les extrémités du cristal en écrivant que le taux de commutation $T_{\mathrm{c}}^{\prime}$ après traversée d'une épaisseur $e$ est :

$$
T_{\mathrm{c}}^{\prime}=T_{\mathrm{c}} \exp ^{-\alpha(\lambda) e} .
$$

$\alpha_{(\lambda)}$ est un coefficient d'absorption moyen, peu différent de $\left(\alpha_{\mathrm{e}} \alpha_{0}\right)^{1 / 2}$ où $\alpha_{0}$ et $\alpha_{\mathrm{e}}$ désignent les coefficients d'absorption respectifs du mode ordinaire et du mode extraordinaire.

Ce coefficient d'absorption croît très vite quand on se rapproche du seuil d'absorption fondamentale e'est-à-dire au-delà de $\lambda_{i}$ du côté des courtes longueurs d'ondes. Il limite le coefficient de transmission effectif d'une structure passe-bande d'épaisseur $e$ à la valeur maximale :

$$
T_{\mathrm{ci}}^{\prime}=\mathrm{e}^{-\alpha_{\left(\lambda_{1}\right) e}} \quad \text { avec } e=(2 p+1) \frac{L_{\mathrm{ci}}}{2} .
$$

A ce point de vue, il y a donc intérêt à choisir $p=0$ plutôt que $p \neq 0$ qui donnerait une meilleure sélectivité, et à diminuer l'absorption résiduelle audessous du gap. Ceci peut être fait en jouant sur la température (phonons), sur la pureté du cristal (absorption par impuretés) ou encore en diminuant la longueur de cohérence isotrope

$$
L_{\mathrm{ci}}=\lambda_{\mathrm{i}} \frac{n}{n_{x y}} \cdot \frac{1}{n_{x y}}
$$

qui décroît très vite quand on accroît le couplage c'est-à-dire la valeur de $n_{x y}$. Ceci peut être obtenu expérimentalement de manière très simple par effet photo-élastique.

2. Dispositif expérimental et cristaux. -2.1 DisPOSITIF EXPÉRIMENTAL. - L'échantillon de 1 à $2 \mathrm{~mm}$ d'épaisseur est placé dans un cryostat à azote liquide

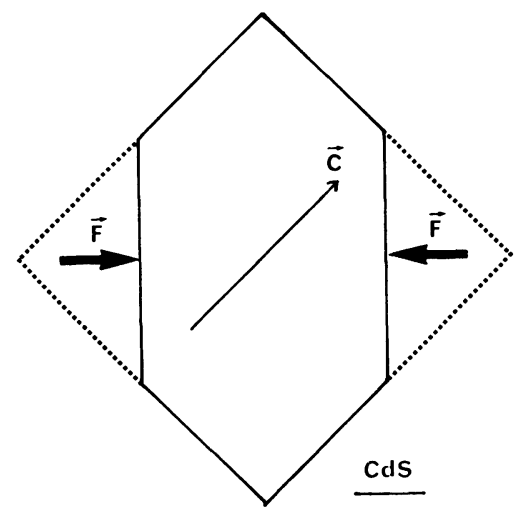

(a)

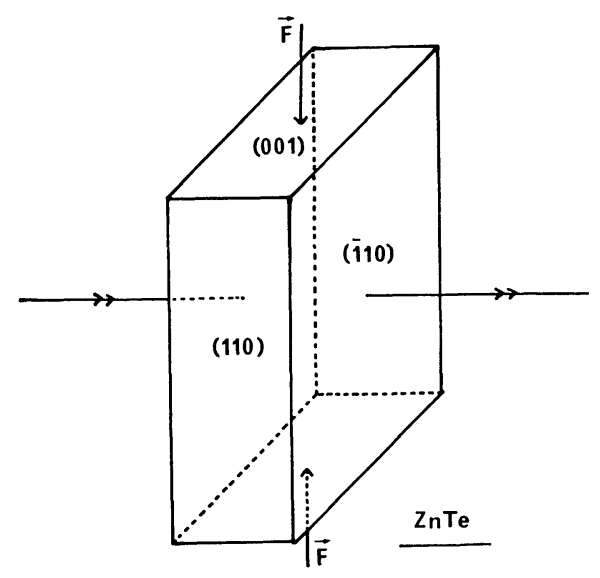

(b)

Fig. 5. - Plan de taille des échañtillons. [Samples configurations.] 
muni de 2 fenêtres optiques et d'un dispositif mécanique permettant d'exercer une contrainte uniaxe. Les polariseurs sont du type glazebrook et la source de lumière est une lampe à Xénon Type XBO $75 \mathrm{~W}$. L'analyse spectrale est faite avec un monochromateur à réseau Jobin-Yvon Type $\mathbf{M} 25$ quand on se contente d'une faible résolution, $30 \mathrm{~cm}^{-1}$, ou grâce à un triple monochromateur CODERG T 800 dont la résolution est supérieure à $1 \mathrm{~cm}^{-1}$.

Les températures utilisées sont $77 \mathrm{~K}$ et $300 \mathrm{~K}$. La contrainte uniaxe sur l'échantillon est ajustée à une valeur telle que l'épaisseur $e$ soit égale à $L_{\mathrm{c}_{\mathrm{i}}} / 2$ : elle est mesurée par un capteur piézo-électrique KISTLER Type 9001. Dans la plupart des cas le phénomène est visible sur les cristaux bruts de tirage et l'ajustement de la pression et de la température ne sert qu'à optimiser les performances.
2.2 Cristaux. - CdS. - Les cristaux étudiés sont de provenance Eagle-Picher, Harshaw (USA) et Techsnabexport (URSS). Ils présentent une biréfringence naturelle de l'ordre de $2 \times 10^{-2}$ pour une longueur d'onde de $0,6 \mu \mathrm{m}$. Ils sont de type semiconducteur $\mathrm{N}$, avec une résistivité d'obscurité de l'ordre de $10 \Omega \mathrm{m}$. Nous avons préféré le CdS semiconducteur au CdS photoconducteur car ce dernier, compensé au $\mathrm{S}$, a un coefficient de transmission nettement plus faible à la longueur d'onde isotrope.

Les échantillons sont d'abord découpés en forme de parallélépipèdes de dimensions $1 \times 5 \times 5 \mathrm{~mm}^{3}$ environ, l'axe optique étant perpendiculaire à une des petites faces. Les faces $5 \times 5 \mathrm{~mm}^{2}$ sont polies sur toile avec une pâte diamantée de $1 \mu \mathrm{m}$. L'échantillon est ensuite retaillé, comme l'indique la figure $5 a$, pour appliquer une contrainte uniaxe à $45^{\circ}$ de l'axe

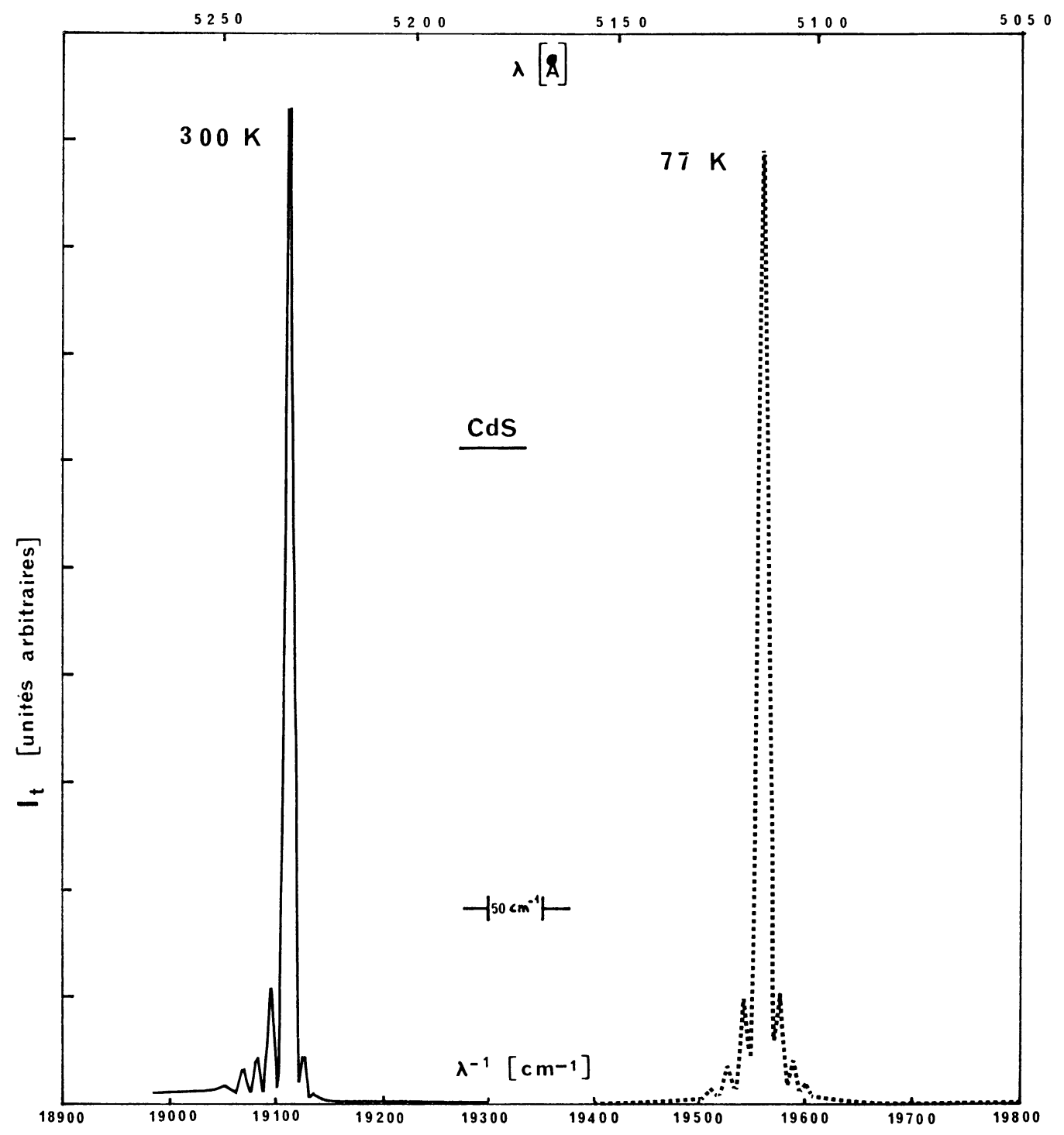

FIG. 6. - Spectre de transmission obtenu sur CdS en structure passe-bande à $300 \mathrm{~K}$ (trait continu) et à $77 \mathrm{~K}$ (trait pointillé). Noter à $300 \mathrm{~K}$ la dissymétrie due à l'absorption. Cette dissymétrie disparaît à $77 \mathrm{~K}$ en même temps que la queue d'absorption résiduelle.

[Transmission peak of a band-pass CdS structure at $300 \mathrm{~K}$ and $77 \mathrm{~K}$. Note that the assymetry due to the absorption is clearly appearent at $300 \mathrm{~K}$ and disappears at $77 \mathrm{~K}$.] 
optique, ce qui permet de contrôler la valeur de l'élément non diagonal $n_{x y}$.

$-\mathrm{CdS}_{1-x} \mathrm{Se}_{x}$ et $\mathrm{Zn}_{1-x} \mathrm{Cd}_{x} \mathrm{~S}$. - Nous avons pu disposer d'une part d'une série de cristaux de $\mathrm{CdS}_{1-x} \mathrm{Se}_{x}$ à composition chimique homogène mais avec des valeurs de $x$ étagées de 5 en $5 \%$ entre 0 et 1 , provenance Dr. D. C. Reynolds (USA).

D'autre part les Drs Ruppel et H. Rufer de l'Université de Karlsrühe ont bien voulu mettre à notre disposition de très beaux cristaux de $\mathrm{CdS}_{1-x} \mathrm{Se}_{x}$ et $\mathrm{Zn}_{1-x} \mathrm{Cd}_{x} \mathrm{~S}$ dans lesquels la composition chimique varie de façon continue sur une distance de l'ordre de 1 à $1,5 \mathrm{~cm}$. Cette composition chimique a été mesurée avec une précision de l'ordre de $1 \%$ à l'aide d'une sonde électronique.

- ZnTe. - Nos mesures ont porté sur des cristaux de provenance LETI, Grenoble, avec la géométrie indiquée dans la figure $5 b$.

La contrainte uniaxe appliquée dans la direction 001 sert à induire une piézo-biréfringence en faisant passer de la structure blende, $T_{d}^{2}$ ou $\overline{4} 3 \mathrm{~m}$ à la structure $\mathrm{D}_{2}^{\mathrm{d}}$ ou $\overline{4} 2 \mathrm{~m}$. Pour des raisons de simplicité, nous avons renoncé à appliquer une seconde contrainte à $45^{\circ}$ de la première pour optimiser les performances et nous nous sommes contentés de vérifier la réalité du transfert d'énergie.

3. Résultats expérimentaux. - 3.1 CRISTAUX STRUCTURE WURTZITE $\mathrm{C}_{6 \mathrm{v}}(6 \mathrm{~mm}) .-3.1 .1$ Coupebandes ou passe-bandes à fréquence fixe. - CdS. - La figure 6 représente l'allure type du spectre de transmission obtenu à $77 \mathrm{~K}$ et $300 \mathrm{~K}$ en structure passebande c'est-à-dire avec polariseur et analyseur croisés.

A $77 \mathrm{~K}$ la largeur à mi-hauteur du pic, centré sur $\lambda_{\mathrm{i}}=5112 \AA\left(19560 \mathrm{~cm}^{-1}\right)$ est de $4 \AA\left(15 \mathrm{~cm}^{-1}\right)$. Le coefficient de transmission au pic peut atteindre 70 à $80 \%$. A $300 \mathrm{~K}$ le pic est centré sur $\lambda_{\mathrm{i}}=5233 \AA$ $\left(19110 \mathrm{~cm}^{-1}\right)$, sa largeur à mi-hauteur est de $5 \AA\left(20 \mathrm{~cm}^{-1}\right)$, mais le coefficient de transmission n'est plus que de l'ordre de 15 à $20 \%$. Il est intéressant de remarquer qu'une température de $138 \mathrm{~K},\left( \pm 2^{\circ}\right)$ permet de se caler sur la longueur d'onde principale du Laser Argon, $5145 \AA$.

- $\mathrm{CdS}_{1-x} \mathrm{Se}$ et $\mathrm{Zn}_{1-x} \mathrm{Cd}_{x} \mathrm{~S}$. - La figure 7 représente les résultats obtenus avec une série d'échantillons de $\mathrm{CdS}_{1-x} \mathrm{Se}_{x}$ dans laquelle la composition chimique $x$ varie de manière quantifiée de 0 à 1 . On obtient bien le glissement escompté de la fréquence d'accord entre $5100 \AA$ et $7100 \AA$. Ces spectres ont été obtenus à $77 \mathrm{~K}$ avec des échantillons différents dont l'épaisseur variait entre $0,45 \mathrm{~mm}$ et $1,2 \mathrm{~mm}$. Les facteurs de transmission obtenus sont en général inférieurs à ceux du CdS pur, mais ils ont été mesurés en l'absence de toute contrainte extérieure, c'est-à-dire que l'épaisseur était certainement différente de la valeur optimale $L_{\mathrm{ci}} / 2$. C'est le cristal de composition $x=0,5$ qui était le plus mince, $0,45 \mathrm{~mm}$, et qui présentait le maximum d'absorption à la longueur

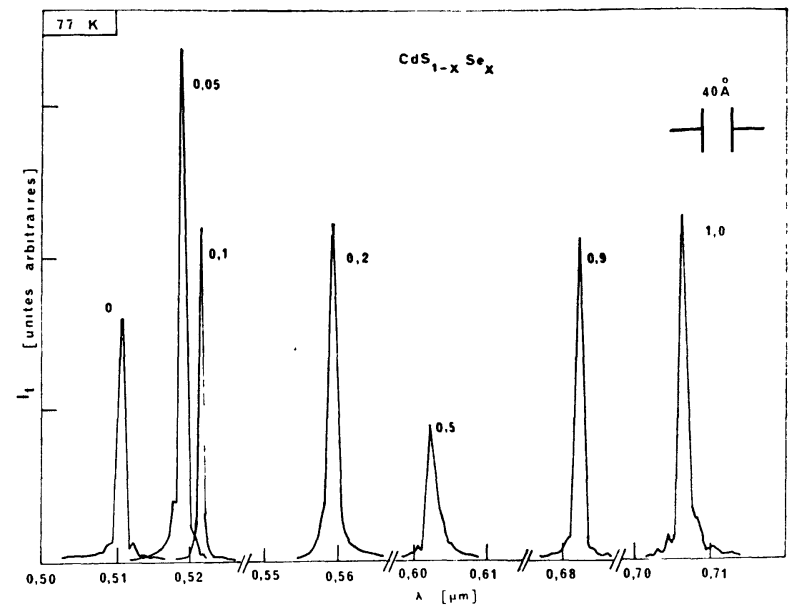

FIG. 7. - Spectres de transmission en structure passe-bande à $77 \mathrm{~K}$ sur des cristaux de $\mathrm{CdS}_{1-x} \mathrm{Se}_{x}$.

[Intensity transfer for $\mathrm{CdS}_{1-x} \mathrm{Se}_{x}$ mixed crystals. Note the discontinuities on the wavelength scale.]

d'onde isotrope. Ceci est vraisemblablement dû à la présence de niveaux d'impureté peu profonds dans cet échantillon.

3.1.2 Coupe-bandes ou passe-bandes accordables. - Avec les cristaux à composition chimique variable de façon continue le phénomène est analogue mais, bien entendu, le glissement de la longueur d'onde d'accord est lui aussi continu. La figure 8 représente

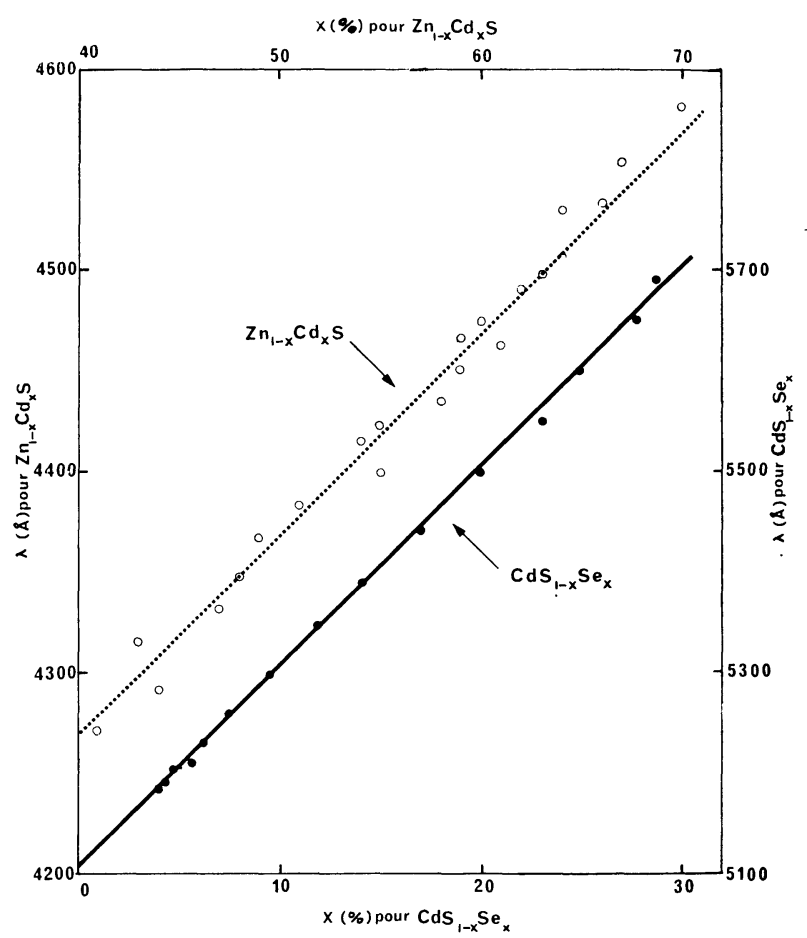

FIG. 8. - Longueur d'onde isotrope en fonction de la composition chimique sur des cristaux de $\mathrm{CdS}_{1-x} \mathrm{Se}_{x}$ et $\mathrm{Zn}_{1_{-} x} \mathrm{Cd}_{x} \mathrm{~S}$ à composition chimique continuement variable (température $77 \mathrm{~K}$ ).

[Wavelength $\lambda_{\mathrm{t}}$ corresponding to the transmission peak as a function of the fractionnal composition $x$ in $\mathrm{CdS}_{1-x} \mathrm{Se}_{x}$ and $\mathrm{Zn}_{1-x} \mathrm{Cd}_{x} \mathrm{~S}$ (Data are taken at $77 \mathrm{~K}$ ).] 
les résultats obtenus avec 2 échantillons bruts de tirage (pas de contrainte uniaxe). Le facteur de transmission au pic est, dans ces conditions, de 15 à $20 \%$ avec une largeur à mi-hauteur de l'ordre de 5 à $10 \AA$.

3.2 Cristaux a structure zinC-Blende $\mathrm{T}_{\mathrm{d}}^{2}(\overline{4} 3 \mathrm{~m})$. - Pour obtenir des filtres de ce type dans l'infrarouge ou l'ultraviolet, il faut utiliser des cristaux dont la bande interdite varie de quelques dizièmes d'électronvolt à quelques électrons-volts. Ceci impose de recourir à des classes cristallines autres que la classe $C_{6 v}$ sous réserve que :

- la biréfringence, naturelle ou induite, s'annule en changeant de signe dans la zone de transparence;

- le couplage entre modes polarisés existe c'est-àdire qu'il y ait un élément non diagonal dans le tenseur de permittivité diélectrique.

La première condition est vérifiée par un grand nombre de cristaux parmi lesquels on peut citer
$\mathrm{MgF}_{2}$ et $\mathrm{Al}_{2} \mathrm{O}_{3}$ côté ultraviolet [6] et les semiconducteurs tels $\mathrm{GaAs}, \mathrm{GaSb}, \mathrm{Ge}, \mathrm{CdTe}$ [7] côté proche infrarouge, avec la structure zinc-blende.

Il était donc important de vérifier que le couplage entre modes E. M. polarisés se produisait également dans cette classe cristalline et c'est ce que nous avons fait en recourant au $\mathrm{ZnTe}$ pour pouvoir utiliser le même appareillage optique qu'avec les semi-conducteurs II-VI. En effet la piézo-biréfringence de ce matériau, induite par une contrainte 001 , s'annule vers $5700 \AA$ à $300 \mathrm{~K}$ [4]. Nous n'avons pas trouvé dans la littérature de résultats publiés sur la biréfringence induite du ZnTe à $77 \mathrm{~K}$ mais, en admettant que la longueur d'onde isotrope suit à peu près la longueur d'onde d'absorption fondamentale, on peut d'après [8] prévoir une valeur de l'ordre de $5450 \AA$.

Les résultats obtenus à $77 \mathrm{~K}$, figure 9 , démontrent clairement que le couplage existe et qu'il y a transfert d'énergie à l'isochronisme. Le facteur de transmission obtenu, $T_{\mathrm{c}}^{\prime} \sim 70 \%$ n'a pas été optimisé par ajuste-

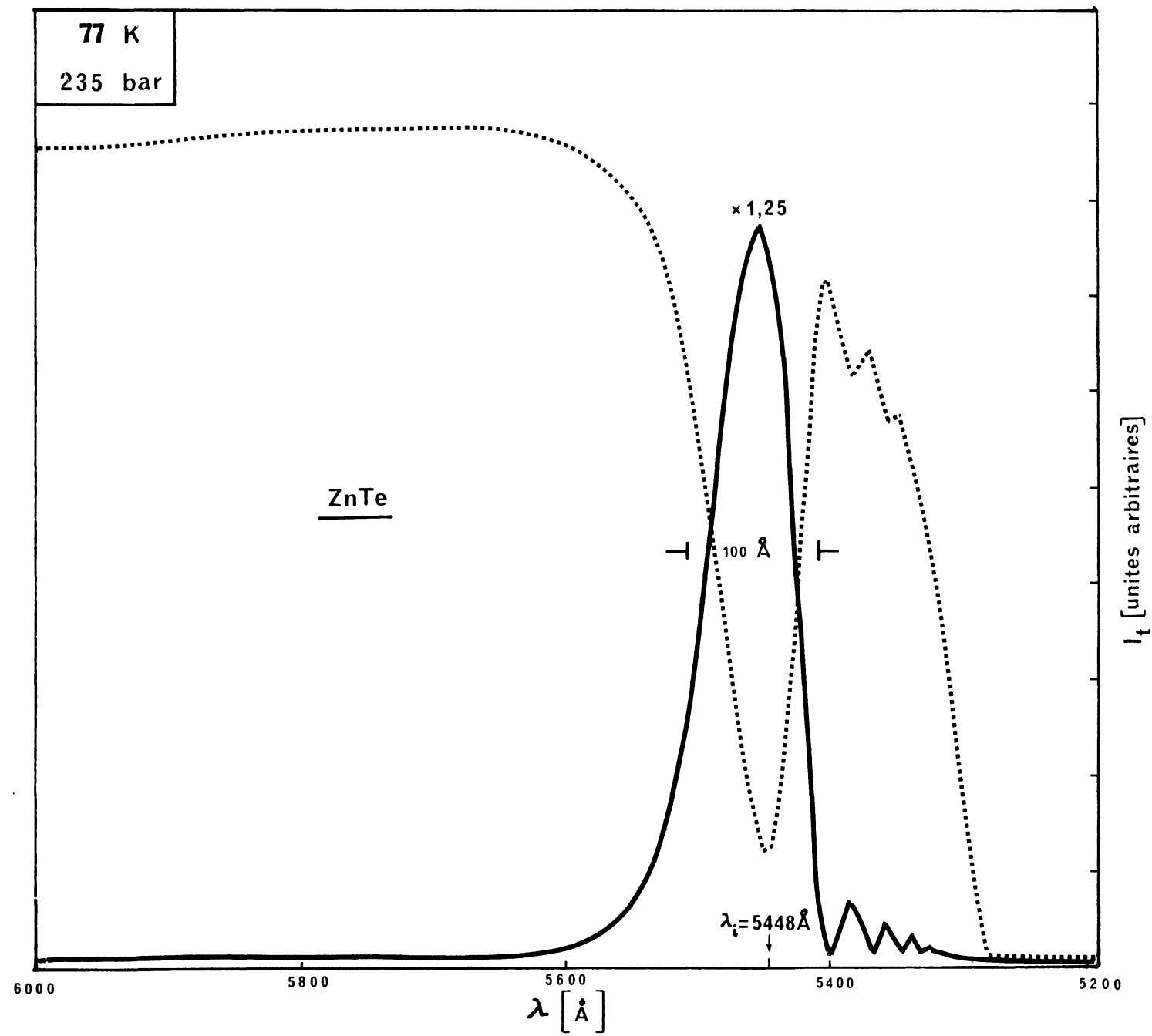

FIG. 9. - Spectres de transmission en structure passe-bande (trait continu) et coupe-bande (trait pointillé) obtenus sur ZnTe à $77 \mathrm{~K}$ en présence d'une contrainte 001 de 235 bar.

[Spectral dependence of the magnitude of the transmitted light throught a $\mathrm{ZnTe}$ crystal at $77 \mathrm{~K}$ with a $<001>$ stress of 235 bar. Full line band-pass structure. Dotted line, cut band structure.] 
ment de la longueur de cohérence, sa valeur n'est donc donnée qu'à titre indicatif et il est certainement possible de faire mieux. Sur cet enregistrement, effectué sous une pression de 235 bar, la largeur du pic à mi-hauteur est de $70 \AA$, c'est-à-dire que la sélectivité est 10 à 20 fois inférieure à celle obtenue avec le CdS. La largeur du pic $\Delta \lambda$ est ici réglable en ajustant la valeur de la pression, puisqu'elle est inversement proportionnelle à la dispersion de piézo-biréfringence autour de $\lambda_{i}$ qui est elle-même proportionnelle à la contrainte. Les expériences faites à 100 bar, 150 bar et 200 bar, donnent effectivement des largeurs à mi-hauteur de $140 \AA, 90 \AA$ et $70 \AA$. Si on admet que l'échantillon peut supporter sans dommage des pressions de l'ordre de $1 \mathrm{kbar}$ [4], on peut espérer obtenir une largeur à mi-hauteur de l'ordre de $15 \AA$.

4. Performances limites d'une structure coupe-bande à CdS en vue d'une application type spectroscopie
Raman (300 K). - Nous avons déjà signalé que la longueur d'onde isotrope $\lambda_{\mathbf{i}}$ du sulfure de cadmium varie entre $5235 \AA$ à $300 \mathrm{~K}$ et $5110 \AA$ à $77 \mathrm{~K}$ c'est-àdire de part et d'autre de la raie principale d'un laser argon $(5145 \AA$ ). Il nous a donc paru intéressant de chiffrer les performances limites d'une structure coupe-bande susceptible d'être utilisée pour éliminer par exemple la raie de diffusion Rayleigh en spectroscopie Raman. Les résultats obtenus à $300 \mathrm{~K}$ avec une seule traversée du cristal sont reportés sur la figure 10.

L'échelle verticale utilisée est une échelle logarithmique ; à $50 \mathrm{~cm}^{-1}$ de la fréquence centrale, côté grandes longueurs d'ondes, c'est-à-dire côté raies stokes, l'affaiblissement résiduel par absorption et réflexion aux extrémités est de $5 \mathrm{db}$ alors que l'affaiblissement sur la fréquence centrale est de $45 \mathrm{db}$. Il y a donc un affaiblissement relatif de $40 \mathrm{db}$.

En utilisant un double passage à travers le cristal

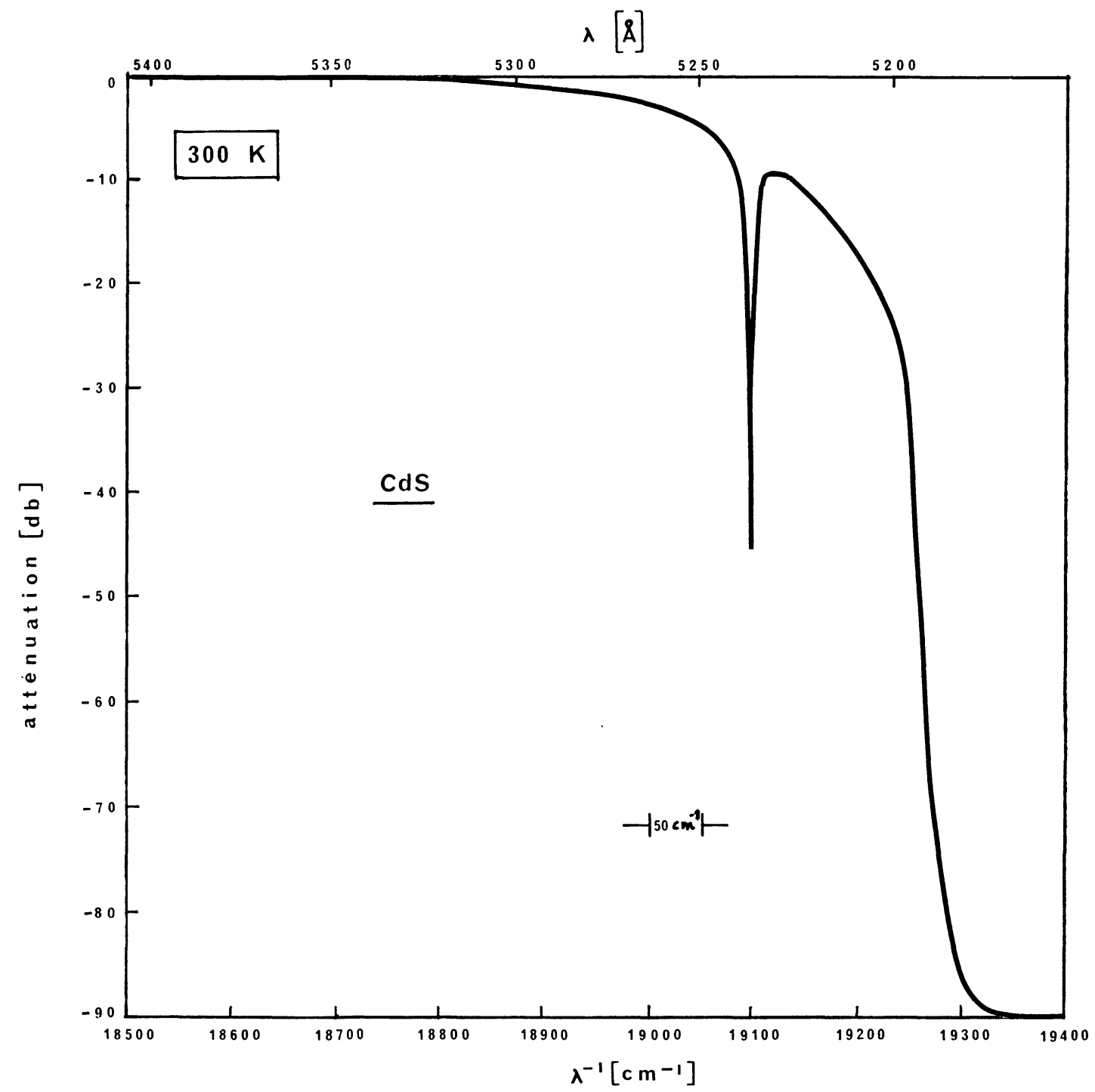

FIG. 10. - Enregistrement en échelle logarithmique d'un spectre de transmission en structure coupe-bande sur $\mathrm{CdS}$ à $300 \mathrm{~K}$ dans le cas d'un couplage optimum (simple passage). [Recorder trace with a logarithmic scale of a cut band structure obtained with a $\mathrm{CdS}$ crystal at $300 \mathrm{~K}$ (one way transit of the light).] 
(en fait 2 cristaux) nous avons obtenu, toujours à $300 \mathrm{~K}$, les résultats représentés sur la figure 11 . L'affaiblissement sur la fréquence centrale est cette fois de $85 \mathrm{db}$ et de $10 \mathrm{db}$ à $50 \mathrm{~cm}^{-1}$ côté grandes longueurs d'onde. Le taux de réjection est donc de $75 \mathrm{db}$. Bien noter que ces résultats n'ont qu'une valeur indicative puisqu'ils ont été obtenus à $300 \mathrm{~K}$ alors que l'accord sur la raie de l'argon, $5145 \AA$, imposerait une température de l'ordre de $138 \mathrm{~K}$. A cette température, l'absorption résiduelle au voisinage du gap serait diminuée de 5 à $6 \mathrm{db}$ ce qui améliorerait sensiblement la transmission hors-bande sans altérer le taux de réjection. L'intérêt réel d'un tel système nous paraît d'ailleurs résider dans l'utilisation conjointe d'une telle structure continuement accordable et d'un laser à colorant.

5. Conclusion. - Nous espérons avoir montré l'intérêt du couplage de modes électromagnétiques polarisés pour obtenir des filtres optiques passe- bande ou coupe-bande à fréquence fixe ou continuement accordable.

Nous avons démontré que ces systèmes sont réalisables dans presque tout le spectre visible en utilisant des cristaux biréfringents à structure Wurtzite.

Nous avons également montré que l'on pouvait recourir à d'autres classes cristallines, structure blende notamment, pour étendre le domaine potentiel d'applications côté proche infra-rouge et ultra-violet.

Les performances de ces filtres sont remarquables à la température de l'azote liquide puisqu'on obtient par exemple avec une structure passe-bande :

- facteur de transmission 60 à $80 \%$,

- largeur au pied $5 \AA$,

- taux de réjection hors bande 50 à $80 \mathrm{db}$,

- très faible sensibilité à l'ouverture du faisceau.

A la température ambiante, le facteur de transmission tombe à 15 ou $20 \%$, les autres caractéristiques étant peu altérées. Il y a donc un effort à faire

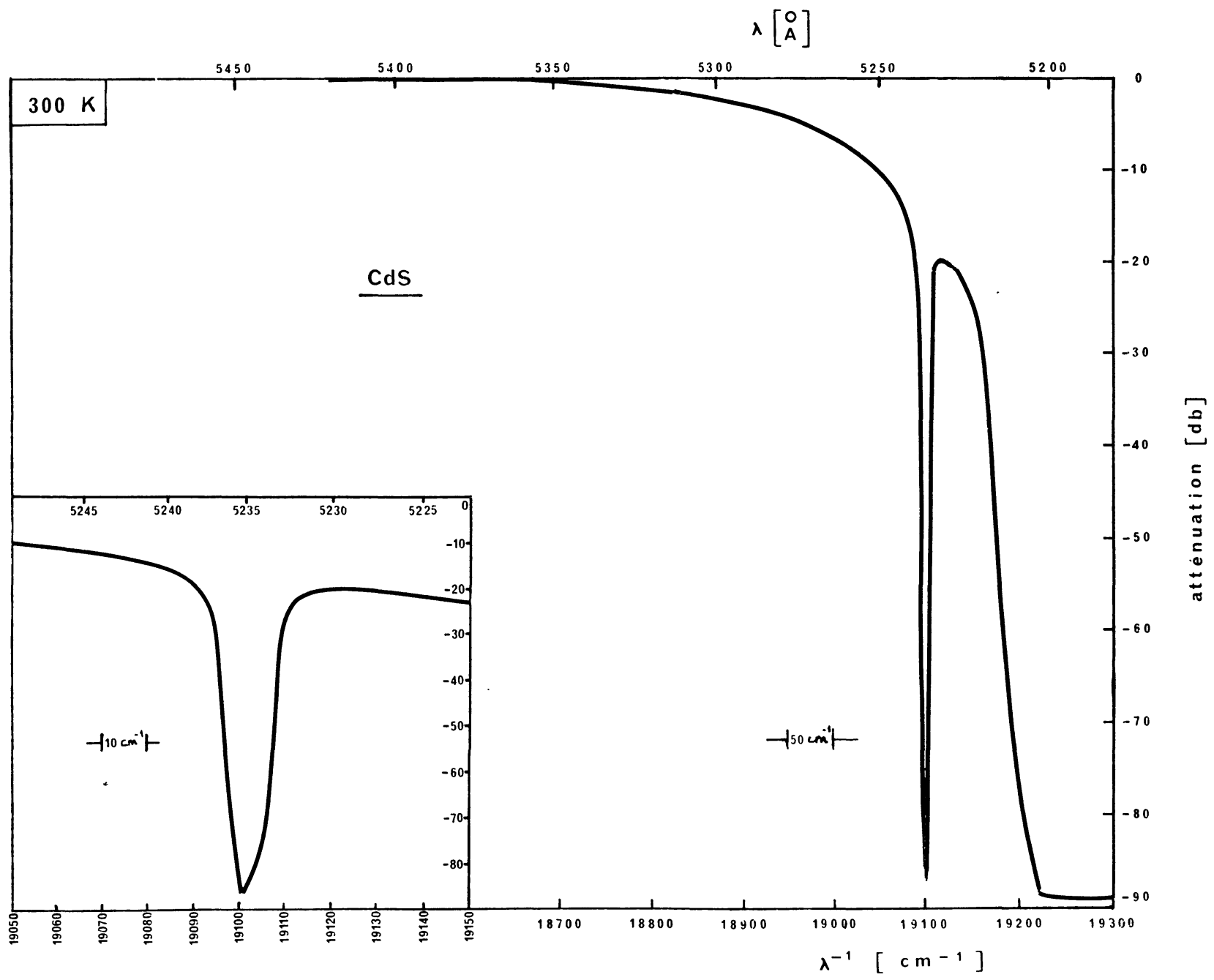

FIG. 11. - Enregistrement en échelle logarithmique d'un spectre de transmission en structure coupe-bande sur CdS à $300 \mathrm{~K}$ dans le cas d'un couplage optimum (double passage). En médaillon, nous avons reproduit avec une échelle de longueur d'ondes dilatée, la portion de spectre située dans la région de la longueur d'onde isotrope.

[Recorder trace with a logarithmic scale of a cut band structure obtained with CdS at $300 \mathrm{~K}$ using a double transit of the light. Insert : details of the cut band behavior in an enlarged wavelength scale.] 
pour la recherche de matériaux présentant une meilleure transparence au voisinage de l'absorption fondamentale à $300 \mathrm{~K}$.

Les 2 conditions impératives pour obtenir le transfert d'énergie entre modes polarisés à angle droit sont :

- l'accord de phase,

- l'existence d'un élément de couplage.

La première de ces conditions est réalisée dans les cristaux dont la biréfringence s'annule en changeant de signe dans une région de transparence.

Le cas n'est pas exceptionnel et la recherche des matériaux adéquats est guidée par les considérations de symétrie qui précisent le caractère permis ou interdit des transitions interbandes suivant l'etat de polarisation du photon.

La condition relative au couplage semble, d'après nos résultats, être généralement satisfaite sans que le mécanisme microscopique exact en soit parfaitement établi. C'est un point que nous nous proposons de discuter dans un prochain article.

Remerciements. - Les auteurs remercient la D. G. R. S. T. pour son soutien financier au cours de cette étude.

\section{Bibliographie}

[1] Henry, C. H., Phys. Rev. 143 (1966) 627-633.

[2] Laurenti, J. P., Rustagi, K. and Rouzeyre, M., Appl. Phys. Lett. 28 (1975) 212-213.

[3] Laurenti, J. P., Rustagi, K. and Rouzeyre, M., J. Apfl. Phys. 48 (1977) 203-204.

[4] Yu, P. Y. and Cardona, M., J. Phys. Chem. Sol. 34 (1973) 29-56.
[5] Czyzak, S. J., Crane, R. C. and Bienewski, T. M., J. Opt. Soc. Amer. 49 (1959) 485-497.

[6] Chandrasekharan, V. and Damany, H., Aprl. Opt. 8 (1969) 671-675.

[7] Yu, P. Y., Cardona, M. and Pollak, F. H., Phys. Rev. B 3 (1971) 340-346.

[8] Camassel, J. and Auvergne, D., Phys. Rev. B 12 (1975) 3258-3267. 